\title{
Does a six-weeks of Hip-focused exercises has the effects on Time to Stabilization after Anterior Cruciate Ligament Reconstruction?
}

\section{Thanathep Tanpowpong}

Chulalongkorn University

Orawan Jaiharn ( $\sim$ orawanj.pt@gmail.com )

Chulalongkorn University https://orcid.org/0000-0003-1904-4647

\section{Original Research Article}

Keywords: Hip-focused exercise, Anterior Cruciate Ligament Reconstruction, Time to Stabilization, Peak torques, Isokinetic

Posted Date: March 15th, 2020

DOl: https://doi.org/10.21203/rs.3.rs-17048/v1

License: (c) (i) This work is licensed under a Creative Commons Attribution 4.0 International License.

Read Full License 


\section{Abstract}

Background: The investigation of hip muscles on knee functions has been broadly published. The deficits in proximal neuromuscular control has been identified as a contributor to valgus knee which is compromise to knee injuries. The aim of study was to determine the relationship between muscle strength and time taking to maintain the stability. Methods: Thirteen male underwent Anterior Cruciate Ligament Reconstruction (ACLR) participated in the six week of hip-focused exercise program. Single leg jump-landing test were performed to determine the Time to Stabilization (TTS). Concentric hip abductionadduction and knee extension-flexion strength was evaluated muscle peak torques at 60 degree/second. Results: The study demonstrated that there was a strength improvement on both hip and knee muscles. Moreover, the IKDC score was significantly increased (82.71 \pm 15.21 to $90.80 \pm 8.44$ ) after completed the exercise. We found no correlation between muscle peak torques and TTS. However, there was a moderate relationship between hip abduction and knee extension strength $(r=0.624, p=0.023)$. Conclusion: The main findings suggesting that rehabilitation program targets on strengthen the hip muscles demonstrate an improvement of knee functions in individual with ACLR.

\section{Introduction}

Most sporting events are related with the multidirectional movements, particularly on the elite level. Many injuries reported on the Anterior Cruciate Ligament (ACL) has been established. ${ }^{(1,2)}$ The study of biomechanical, therefore has become an important issue, both on injury prevention and rehabilitation. Dynamic postural control has been addressed to be a measurement goal before return to play. ${ }^{(3-5)}$ However, there is a study reveals that patients with history of average 2.5 years following ACLR, has a longer time to control the stability after a jumped test. ${ }^{(6)}$ Although the important of Quadriceps and Hamstrings muscle has been well documented on knee stability functions. Prolong time to recover is prominent in the group of ACL deficient knee. ${ }^{(7)}$ The investigating on hip muscle activation during stance time has suggested that the moment of hip abductors, extensors and external rotators are increased to preserve body's balance. ${ }^{(8)}$ Weakness of the hip muscle group has also identified as a contributor to valgus knee. ${ }^{(9)}$ Previous studies has extensively published on the relationships between the deficiencies of proximal neuromuscular control on knee injuries. ${ }^{(10-13)}$ These suggesting that applying the rehabilitation exercise which target on hip muscle could lead to improvement of functional results. Furthermore, a review study of Ford KR and colleagues has demonstrated that the exercises focused on hip muscle groups had contribute to strengthen the muscle activations. ${ }^{(14)}$ Therefore, the purpose of this study was to examine whether there is a correlation between muscle strength and TTS following a hipfocused exercise. We hypothesized that increasing in strength of muscle would demonstrated the stronger relationship on TTS in patient with ACLR.

\section{Method}


Thirteen active male with the history of Anterior Cruciate Ligament Reconstruction (ACLR) between 1-3 years volunteered to the study. Among all participants were implicated in several kind of sports (e.g. running, rugby, football, basketball, badminton and hockey). Any other previously serious knee pain and/or surgery, limiting of knee range of motion were excluded. Written informed consent approving by The Ethics Committee of faculty of Medicine, Chulalongkorn University was obtained from all participants.

The testing process consisted of muscle strength test using an isokinetic dynamometer (Humac Norm 2009), Single-leg jump landing test performing on a force platform (Bertec FP4060-07-1000, Sampling rate $1,000 \mathrm{~Hz}$ ) and the self-reporting of The International Knee Documentation Committee (IKDC) were used to evaluate knee functions. The Hip-focused exercises was initiated within a week after the pretest. The process was considered to be completely finished after six week of the exercise.

The single leg jump-landing test was performed totally for five successful trials with one minute rest. Participants were instructed to stand, wore the athletic shoes, at a $70 \mathrm{~cm}$ point away from the center of platform. The testing instructions was to jump anteriorly with both legs, hit the target tab, setting at $50 \%$ of their maximal jump height ${ }^{(6)}$ and firmly land with the reconstructed leg. After touched on the platform, they were asked to remain standing still for 10 second. Any hopping, stepping, missing the target was rejected. To determine the time taking to stabilize after landing, baseline standing values was measured from participants' reconstructed leg, on the force platform for 10 second. The first impact was set at force $>10 \mathrm{~N}$. The maximal point of ground reaction force (GRF) and the decline of GRF to baseline values was used to calculate.

The muscle strength test; hip abductors-adductors and knee extensors-flexors group, was performed on the reconstructed leg. The hip abduction -adduction was conducted in standing position with the strap around the hip level. Whereas, the knee extension- flexion was tested in sitting position. (Figure 1) Testing orders were randomly provided, setting on the concentric contraction mode at speed of 60 degree/second. Warm-up and stretching was ensured before performing three practices with 15 second rest. The maximal five repetitions totally for two trials, separated by two minute rest, were encouraged, verbally, to the participants in order to complete the test. A highest peak torque (Nm) value over two trials was averaged and normalized to participant's body weight $(\mathrm{Nm} / \mathrm{Kg})$ for a statistical analysis using SPSS version 23 for window. Student paired t-test was conducted for the differences. Isokinetic peak torques and time to stabilization was examined using the Pearson's correlation coefficient. The level of significant was set at $p<0.05$

Insert here: Figure 1A the hip abduction-adduction strength testing in standing position,1B the knee extension-flexion strength testing in sitting position

A Hip-focused exercises program consists of five exercises which focus on the activation of hip muscles; clam-shell exercise, side-lying hip abduction, single-leg squat, front plank with hip extension and side plank with hip abduction. (Figure 2) The program was scheduled three times a week for totally six week. 
Participants sustained overall three set of each exercise, performing on both legs, $10-12$ times with two minute rest between set.

Insert here: Figure 2 the hip-focused exercises program

\section{Results}

The participant's characteristic are displayed in table 1. There were no significant difference of TTS after jumped-landing. However, a self-reporting IKDC score was shown the significantly increase after the week sixth of hip-focused exercise. (Table 2)

Insert here: Table 1. The demographic data of the participants (mean $\pm S D$ )

Insert here: Table 2. The mean values of time to stabilization and IKDC score before and after exercise (mean $\pm S D)$

Insert here: Table 3. The mean values of muscle peak torque at speed of 60 degree/second. (mean \pm SD)

The results demonstrated that after overall six week of exercise, there was a significantly increase in absolute peak torque $(\mathrm{p}<0.05)$ for all muscle strength measurements except hip adduction. Converting to percent strength changed, there was improvement by $22.67 \%, 13.88 \%, 23.94 \%$, and $18.45 \%$ for hip abduction, hip adduction, and knee extension and knee flexion, respectively.

Insert here: Table 4. The correlation between muscle peak torque and jumped-landing time to stabilization.

A Pearson's correlation demonstrated no relationships between all muscle peak torques and the time to stabilization after landing on a force platform (Table 4). However, there was a positively moderate relationships between the hip abduction and knee extension strength $(r=0.624, p=0.023)$. These suggested that both hip and knee muscle group are important to promote the stabilization of lower limb. Together with their main actions, these muscle groups are crucial for supporting the balance of hip joint. Additionally, they also has a benefit to counteraction the rotational force, which increasing load on knee joint during the stance time.

\section{Discussion}

The major findings of this study demonstrated that after six weeks of hip-focused exercises, there was strength improvement for both hip and knee muscle groups. Also, the patients' perception regained on their knee functions. However, our main hypothesis has been rejecting since there was no significant difference of the stabilization time.

The mechanism of neuromuscular training has been well documented. ${ }^{(15,16)}$ Jonathan D. Chappell suggests that the exercise targeting on core strength training, balance training and plyometric training 
has impacted on the kinetics and kinematic throughout movements cycle. The study by Christopher Nagelli ${ }^{(17)}$ also suggested that patient with ACLR committing in the neuromuscular training demonstrates a positive changes on kinematics pattern and had diminishing of vertical ground reaction forces following the jump test. In agreement with the study of Hwang-Bo Kak ${ }^{(18)}$, the results has been shown that exercise of Gluteus medius muscle for totally eight weeks increased knee extension strength by $40 \%$ from baseline. Moreover, the study by Bohdanna T. et al ${ }^{(19)}$ has suggested that the neuromuscular training on trunk control may lead to diminish the excessive adduction and internal rotation of leg range of motion during the weight bearing activities. However, our study did not investigate on kinematics including trunk biomechanics. This is considered to be one of the limitation.

In the present study, surprisingly, we founded that increased in hip and knee muscle peak torques but not decreased on the stabilization time. According to the hip-focused exercise program, the position, mostly, is a non-weight bearing exercise based. On the other hand, a test for a time employing to stabilize was set into the weight bearing. Furthermore, the several of sports committing by participants that included in this study might particularly had effects.

The study by Tina Y. Claiborne ${ }^{(9)}$ have demonstrated that the strength of hip muscles is the key role in providing knee stabilization. Especially, the hip abductor is an essential muscle to maintain femur into the neutral line. Suggesting that lower in joint internal moments may compromise to knee injuries. In addition, they also suggested that those with stronger hip abductors and external rotators muscle resulting in less knee joint impairments. Jumped-landing test was employed to the study has been shown to be an easily clinical examination for patients who had knee injuries. The test requires to balance the position proposing that the coordination of muscle is one of an important part regarding to kinetic chain principle. However, the various procedure used to access stability could display on the different results. Angela t. gordon et al. ${ }^{(20)}$ studied on the relations of muscle strength and stability, using the star excursion balance test. The results has been shown that both core and hip muscle has no correlation with the lower limb balance. Inconsistent with the study of Won-Hah Park et al. ${ }^{(21)}$ demonstrated that patients with injury of ACL were shown moderately relationships between knee muscle peak torques and dynamic postural stability prior to surgery. The present study, however found no relationships between time taken to stabilize and muscle peak torques. The possible explanations is the muscle strength measurements were achieved from the isolated muscle testing while the stabilization time was derived via the functional motions. In addition to another study limitation, we only measured strength on the reconstructed limb. In fact, to control body's movement, specifically on sports or exercise skills, the collaboration of several muscle groups, joints as well as a neuromuscular control system are involved.

\section{Conclusion}

ACL reconstruction has provided a successful outcome to regain a mechanical knee joint stability. Yet, the patient with ACLR had changed on both kinetics and kinematics. An impairment on muscle strength 
is likely to be an adjustable component to achieve the safely return to play. The findings reveal that the hip-focused exercise may have benefits on those who associate with multidirectional movements.

\section{List Of Abbreviations}

TTS: Time to Stabilization

ACL: Anterior Cruciate Ligament

ACLR: Anterior Cruciate Ligament Reconstruction

IKDC: the International Knee Documentation Committee

GRF: Ground Reaction Force

\section{Declarations}

\section{Ethics approval and consent to participate}

Written informed consent approving by The Ethics Committee of faculty of Medicine, Chulalongkorn University was obtained from all participants.

\section{Consent for publication}

Not applicable

\section{Availability of supporting data}

Not applicable

\section{Competing interests}

Not applicable

\section{Funding}

This study was supported by Ratchadapiseksompotch Fund, Faculty of Medicine, Chulalongkorn University. Grant number RA61/034.

\section{Authors' contributions}




\section{Acknowledgements}

The authors acknowledge to Sports Medicine laboratory, Faculty of Medicine, Chulalongkorn University.

\section{Authors' information}

Not applicable

\section{References}

1.Lyman S, Koulouvaris P, Sherman S, Do H, Mandl LA, Marx RG. Epidemiology of anterior cruciate ligament reconstruction: trends, readmissions, and subsequent knee surgery. The Journal of bone and joint surgery American volume. 2009;91(10):2321-8.

2.Mall NA, Chalmers PN, Moric M, Tanaka MJ, Cole BJ, Bach BR, Jr., et al. Incidence and trends of anterior cruciate ligament reconstruction in the United States. The American journal of sports medicine. 2014;42(10):2363-70.

3.Culvenor AG, Alexander BC, Clark RA, Collins NJ, Ageberg E, Morris HG, et al. Dynamic Single-Leg Postural Control Is Impaired Bilaterally Following Anterior Cruciate Ligament Reconstruction: Implications for Reinjury Risk. The Journal of orthopaedic and sports physical therapy. 2016;46(5):357-64.

4.A. Zouita Ben Moussa, S. Zouita, C. Dziri, Salah. FZB. Single-leg assessment of postural stability and knee functional outcome two years after anterior cruciate ligament reconstruction. Annals of physical and rehabilitation medicine. 2009;52(6):475-84.

5.Henriksson M., Ledin T., Lars G. Postural control after anterior cruciate ligament reconstruction and functional rehabilitation The American journal of sports medicine. 2001;29(3):359-66.

6. Webster KA, Gribble. PA. Time to Stabilization of Anterior Cruciate Ligament-reconstructed Versus Healthy knees in National Collegiate Athletic Association Division I Female Athletes. Journal of Athletic Training. 2010;45(6):580-5.

7.Ardern CL, Taylor NF, Feller JA, Whitehead TS, Webster KE. Sports participation 2 years after anterior cruciate ligament reconstruction in athletes who had not returned to sport at 1 year: a prospective followup of physical function and psychological factors in 122 athletes. The American journal of sports medicine. 2015;43(4):848-56.

8.Paul D, Tibor H, B. J. Gait biomechanics are not normal after anterior cruciate ligament reconstruction and accelerated rehabilitation. American College of Spons Medicine. 1998. 
9.Tina LC, Charles WA, Varsha G, MP. D. Relationship Between Hip and Knee Strength and Knee Valgus During a Single Leg Squat. Journal of Applied Biomechanics. 2006;22:41-50.

10.Mark VP, Kevin RF, Gregory DM, Rachel H, Timothy EH. Limb Asymmetries in Landing and Jumping 2 Years Following Anterior Cruciate Ligament Reconstruction. Clin J Sport Med. 2007;17(4):258-62.

11. Hidetomo S, Go O, Daisuke U, Katsutoshi N, Naoto E. The influence of hip strength on knee kinematics during a single-legged medial drop landing among competitive collegiate basketball players. International Journal of Sports Physical Therapy. 2015;10(5):592-601.

12.Paterno MV, Schmitt LC, Ford KR, Rauh MJ, Myer GD, Huang B, et al. Biomechanical measures during landing and postural stability predict second anterior cruciate ligament injury after anterior cruciate ligament reconstruction and return to sport. The American journal of sports medicine. 2010;38(10):196878.

13.Stearns KM, Powers CM. Improvements in hip muscle performance result in increased use of the hip extensors and abductors during a landing task. The American journal of sports medicine. 2014;42(3):602-9.

14.Ford KR, Nguyen AD, Dischiavi SL, Hegedus EJ, Zuk EF, Taylor JB. An evidence-based review of hipfocused neuromuscular exercise interventions to address dynamic lower extremity valgus. Open access journal of sports medicine. 2015;6:291-303.

15.Jae-Kwang S, Ho-Suk C, S J-H. Effects of neuromuscular training on knee joint stability after anterior cruciate ligament reconstruction. J Phys Ther Sci. 2015;27.

16.Richard KS, Sangeetha M, Emy G, Jennifer L, Ben P, Sara S, et al. Neuromuscular Control of the Knee During a Resisted Single-Limb Squat Exercise. The American journal of sports medicine. 2005;33(10):1520-6.

17.Christopher N, Samuel W, Stephanie DS, Joshua H, EH T. Biomechanical Deficits at the Hip in Athletes With ACL Reconstruction Are Ameliorated With Neuromuscular Training. The American journal of sports medicine. 2018;46(11):2772-9.

18.Hwang-Bo K, Sun-Ja P, P B-J. The effect of hip abductor exercise on muscle strength and trunk stability after an injury of the lower extremities. J Phys Ther Sci. 2016;28:932-5.

19.Bohdanna T. Z, Timothy EH, Reeves NP, Barry G, C J. Deficits in Neuromuscular Control of the Trunk Predict Knee Injury Risk. The American journal of sports medicine. 2007;35(7):1123-30.

20.Angela TG, Jatin PA, VC S. Relationships between core strength, hip external rotator muscle strength, and star excursion balance test performance in female lacrosse players. International Journal of Sports Physical Therapy. 2013;8(2). 
21.Won-Hah P, Do-Kyung K, Jae Chul Y, Yong Seuk L, Ji-Hye H, Moon Jong C, et al. Correlation between dynamic postural stability and muscle strength, anterior instability, and knee scale in anterior cruciate ligament deWcient knees. Arch Orthop Trauma Surg. 2010;130:1013-8.

\section{Tables}

Due to technical limitations, all tables are only available for download from the Supplementary Files section.

\section{Figures}
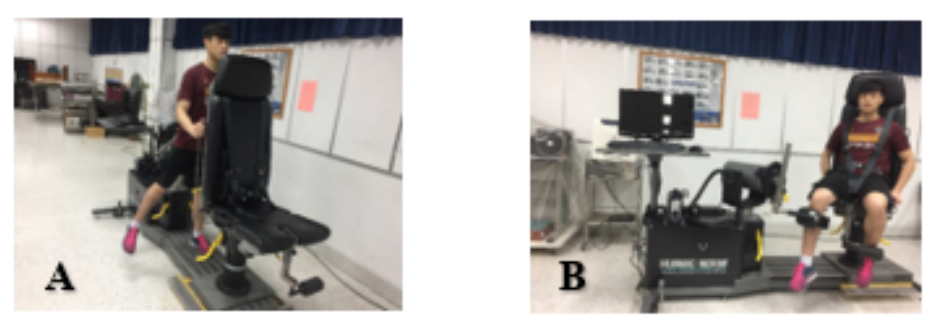

\section{Figure 1}

A) the hip abduction-adduction strength testing in standing position, B) the knee extension-flexion strength testing in sitting position
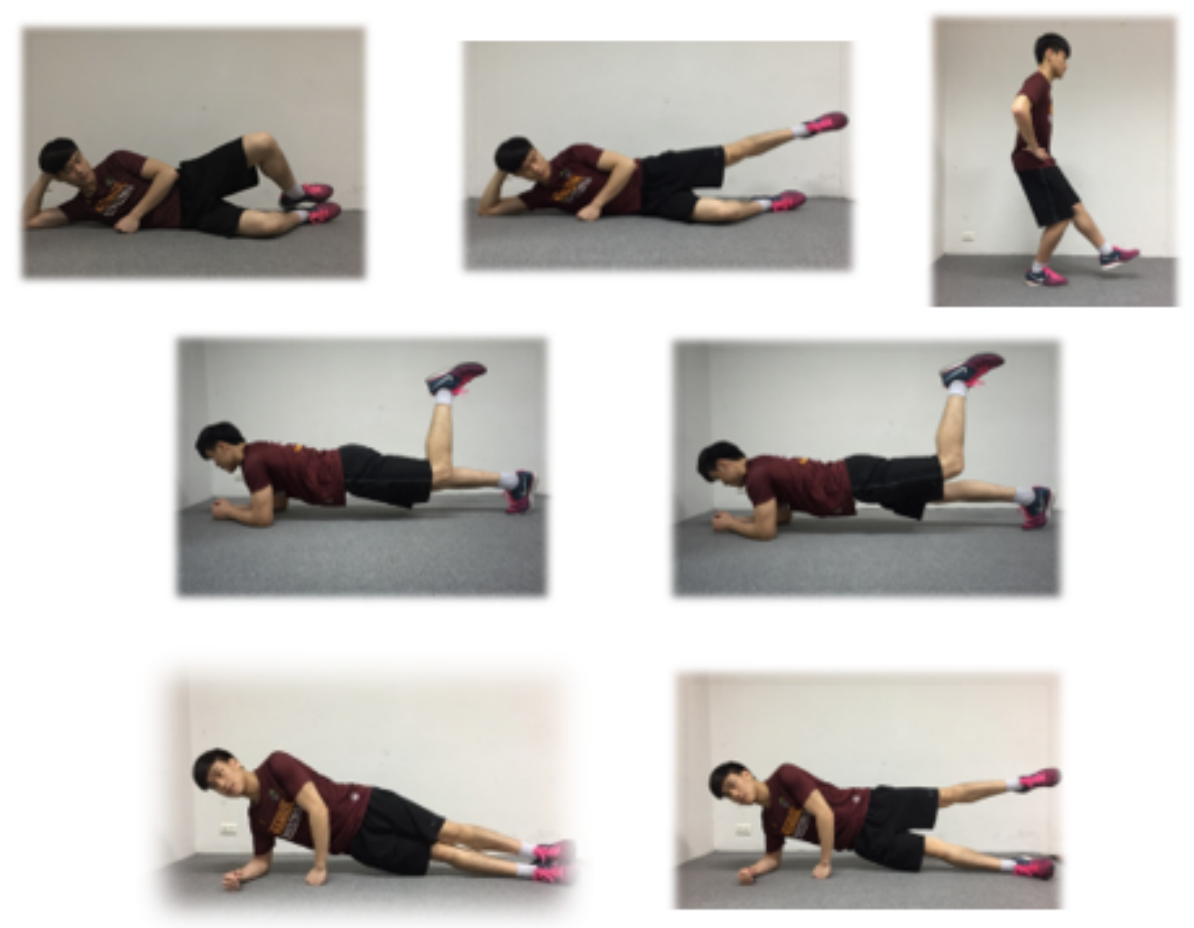

\section{Figure 2}

the hip-focused exercises program 


\section{Supplementary Files}

This is a list of supplementary files associated with this preprint. Click to download.

- Tables.docx 